\title{
ALCAM predicts future cardiovascular death in acute coronary syndromes: Insights from the PLATO trial
}

\author{
Thor Ueland ${ }^{\mathrm{a}, \mathrm{b}, \mathrm{c}, *}$, Axel Åkerblom ${ }^{\mathrm{d}, \mathrm{e}}$, Tatevik Ghukasyan ${ }^{\mathrm{e}}$, Annika E. Michelsen ${ }^{\mathrm{a}}$, \\ Richard C. Becker ${ }^{\mathrm{g}}$, Maria Bertilsson ${ }^{\mathrm{e}}$, Andrzej Budaj ${ }^{\mathrm{h}}$, Jan H. Cornel ${ }^{\mathrm{i}}$, Anders Himmelmann, \\ Stefan K. James ${ }^{\mathrm{d}, \mathrm{e}}$, Agneta Siegbahn ${ }^{\mathrm{e}, \mathrm{k}}$, Robert F. Storey ${ }^{1}$, Frederic Kontny ${ }^{\mathrm{m}, \mathrm{n}}$, Pål Aukrust $\mathrm{t}^{\mathrm{a}, \mathrm{b}, \mathrm{c}, \mathrm{f}}$, \\ Lars Wallentin ${ }^{\mathrm{d}, \mathrm{e}}$, for the PLATO Investigators
}

${ }^{a}$ Research Institute of Internal Medicine, The National Hospital, University of Oslo, Oslo, Norway

${ }^{\mathrm{b}}$ K.G. Jebsen Inflammatory Research Center, University of Oslo, Norway

${ }^{c}$ K.G. Jebsen - Thrombosis Research and Expertise Center (TREC), University of Troms $\phi$, Troms $\phi$, Norway

${ }^{\mathrm{d}}$ Department of Medical Sciences, Cardiology, Uppsala University, Uppsala, Sweden

${ }^{\mathrm{e}}$ Uppsala Clinical Research Center, Uppsala University, Uppsala, Sweden

${ }^{\mathrm{f}}$ Section of Clinical Immunology and Infectious Diseases, Oslo University Hospital, Rikshospitalet, Oslo, Norway

${ }^{\mathrm{g}}$ Division of Cardiovascular Health and Disease, Heart, Lung and Vascular Institute, University of Cincinnati College of Medicine, Cincinnati, OH, USA

${ }^{\text {h }}$ Postgraduate Medical School, Grochowski Hospital, Warsaw, Poland

i Department of Cardiology, Noordwest Ziekenhuisgroep, Alkmaar, the Netherlands

${ }^{\mathrm{j}}$ AstraZeneca Research and Development, Gothenburg, Sweden

${ }^{\mathrm{k}}$ Department of Medical Sciences, Clinical Chemistry, Uppsala University, Uppsala, Sweden

${ }^{1}$ Department of Infection, Immunity and Cardiovascular Disease, University of Sheffield, Sheffield, United Kingdom

${ }^{\mathrm{m}}$ Stavanger University Hospital, Department of Cardiology, Stavanger, Norway

${ }^{\mathrm{n}}$ Drammen Heart Center, Drammen, Norway

\section{H I G H L I G H T S}

- In 5165 patients with acute coronary syndromes, ALCAM was independently associated with adverse outcome including CV death.

- Circulating ALCAM may be useful in identifying high-risk patients who benefit from more intense secondary prevention measures.

- The role of ALCAM and the associated underlying processes during post-MI remodeling merits further investigation.

A R T I C L E I N F O

\section{Keywords:}

Activated leukocyte cell adhesion molecule

Prognosis

Acute coronary syndromes

$\mathrm{CV}$ death

\begin{abstract}
A B S T R A C T
Background and aims: Activated leukocyte cell adhesion molecule (ALCAM) is upregulated during inflammation and involved in transmigration of leukocytes and T-cell activation. We hypothesized that ALCAM might be associated with recurrent events in patients with acute coronary syndromes (ACS).

Methods: ALCAM was measured in serum obtained on admission, at discharge, 1 month and 6 months in a subgroup of 5165 patients admitted with ACS and included in the PLATelet inhibition and patient Outcomes (PLATO) trial (NCT00391872). The association between ALCAM and the composite endpoint and its components, including cardiovascular (CV) death, non-procedural spontaneous myocardial infarction (MI) or stroke during 1-year follow-up, was assessed by Cox proportional hazards models with incremental addition of clinical risk factors and biomarkers (including high-sensitivity troponin $\mathrm{T}, \mathrm{N}$-terminal pro-B-type natriuretic peptide and growth diff factor-15). Results: The median (Q1-Q3) concentration of ALCAM at admission was 97 (80-116) ng/mL. A 50\% higher level of ALCAM on admission was associated with a hazard ratio (HR) of 1.16 (95\% confidence interval [1.00-1.34] $p=0.043$ ) for the composite endpoint in fully adjusted analysis, mainly driven by the association with CV death (HR 1.45 [1.16-1.82] $p=0.0012$ ).

Conclusions: In patients with ACS, admission level of ALCAM was independently associated with adverse outcome including $\mathrm{CV}$ death even after adjustment for established inflammatory and cardiac biomarkers.
\end{abstract}

\footnotetext{
* Corresponding author. Research Institute of Internal Medicine, Oslo University Hospital, Rikshospitalet, Sognsvannsveien 20 , 0372 Oslo, Norway.

E-mail address: thor.ueland@medisin.uio.no (T. Ueland).
} 


\section{Introduction}

Following plaque rupture and myocardial infarction (MI), cell adhesion molecules (CAMs) are involved in attraction and recruitment of leukocytes into the site of plaque destabilization and tissue damage, promoting trans-endothelial migration that involves coordinated events between inflamed endothelium and activated leukocytes [1,2]. These events could be both protective (e.g., stimulating repair processes) and harmful (e.g. enhance tissue damage and further plaque destabilization). Proteolytic cleavage of the extracellular portion of CAMs from activated cell surfaces lead to soluble isoforms that can be detected in the circulation. Systemic levels of several CAMs correlate with disease severity and outcome in various cardiovascular disorders [2,3]. Thus, high levels of soluble intracellular adhesion molecule -1 , vascular cell adhesion molecule-1 and P-selectin have been detected in patients with acute coronary syndromes (ACS) and are associated with poor prognosis [4-6].

Activated leukocyte cell adhesion molecule (ALCAM), also denoted CD166, belongs to the immunoglobulin gene superfamily and was originally identified as a transmembrane receptor involved in T-cell activation $[7,8]$. ALCAM is also expressed on cardiomyocytes and used as a surface marker for enrichment of these from human embryonic stem cells [9]. ALCAM is markedly upregulated during inflammation and is involved in transmigration of leukocytes across CNS endothelium [10]. We detected consistently higher serum levels of ALCAM in the first days following acute ischemic stroke in patients who subsequently suffered an adverse event and serum ALCAM remained an independent predictor of outcome in adjusted analysis [11]. However, there are currently no data on ALCAM in relation to ACS.

Based on its role inflammation including transmigration of leukocytes into inflamed tissue, we hypothesized that ALCAM might be associated with the risk of new events following ACS. The PLATelet inhibition and patient Outcomes (PLATO) trial encompassed a broad ACS population and proved ticagrelor to be superior to clopidogrel in reducing the composite endpoint of cardiovascular (CV) mortality, MI, or stroke $[12,13]$. In the current PLATO sub-study, we evaluated serum ALCAM levels in a subgroup with blood sampling on admission and if available also at outpatient visits during 6 months follow-up after ACS, together with important prognostic biomarkers, in relation to the composite endpoint of CV death, spontaneous MI and stroke [12,13]. We also explored changes in ALCAM concentrations from admission through 6 months follow up and evaluated modifying effects of ticagrelor on ALCAM levels.

\section{Patients and methods}

\subsection{Design and study population}

The randomized, placebo-controlled PLATO trial (NCT00391872) included a total of 18,624 patients with ACS $[12,13]$. The patients presented with either ST-elevation ACS or non ST-elevation ACS and were randomized to either clopidogrel or ticagrelor treatment in addition to optimal medical therapy, including aspirin, and optional invasive therapy $[12,13]$. Patients were recruited between October 2006 and July 2008 and were followed for up to 12 months after ACS. Venous blood samples were obtained from all patients at randomization as part of the main study. In addition, there was a predefined sub study with serial blood sampling conducted at selected sites aiming to obtain samples from 4000 patients at discharge and after 1 month and from at least 3000 of these patients also at 6 months [12,13]. All patients at these selected sites were continuously invited to the sub study and inclusion of new patients proceeded until it was estimated that at least 3000 patients would be available for blood sampling at 6 months. Patients with a blood sample at baseline and at least one additional blood sample during follow-up were eligible for inclusion in the current analyses. The overall aims of the biomarker sub study program have previously been published $[12,13]$. The study adhered to the Declaration of Helsinki, the research protocol was approved by national and institutional regulatory and ethics committees, and written informed consent was obtained from the patients.

\subsection{Endpoint definition and follow up}

The pre-specified primary endpoint of the present sub study was the composite of CV death (defined as any CV cause of death, sudden death or any death with no clear attributable non-cardiovascular cause), spontaneous MI (defined as non-procedure related, non-fatal, MI type 1 [14], or stroke within one year of follow up [12]. The components of the composite endpoint were also evaluated separately. All endpoints in the PLATO trial were centrally adjudicated by an independent and blinded clinical events adjudication committee, comprising cardiologists or neurologists, in order to subclassify causes of death and to subdivide types of MIs, stroke and bleeding events [12,14].

\subsection{Sampling and laboratory analysis}

Baseline venous blood samples were obtained within $24 \mathrm{~h}$ of admission, prior to the administration of study medication. The venous blood was allowed to clot and serum centrifuged, isolated and frozen in aliquots and stored at $-70{ }^{\circ} \mathrm{C}$ in a central repository in Uppsala Biobank until biochemical analyses were performed. ALCAM concentrations were determined by enzyme-linked immunoassay (RnDsystems, Stillwater, MN) with intra- and inter-assay coefficients of variation $<10 \%$. Hs-TnT, NT-proBNP and cystatin C were determined with sandwich immunoassays on the Cobas $^{\circledR}$ Analytics e601 Immunoanalyzer (Roche Diagnostics, Mannheim, Germany). Total and differential (i.e., neutrophils, lymphocytes and monocytes) white blood cell (WBC) counts and high-sensitivity C-reactive protein (hs-CRP) were analyzed at the UCR laboratory, Uppsala, Sweden, with a spectrophotometric analysis (Architect, Abbott, IL, USA). GDF-15 was measured with a pre-commercial assay (Roche Diagnostics) using a monoclonal mouse antibody for capture and a monoclonal mouse antibody fragment for detection in a sandwich assay format. The results of these analyses in relation to outcomes and effects of study treatment has previously been reported [15-17]. We also include data on Fibrin D dimer, sCD40L, P-selectin, IL-6, IL-10, IL-18, oxidized LDL (oxLDL), ApoA1 and ApoB [18-20].

\subsection{Evaluation of ALCAM expression from public databases}

Data used for the analyses of ALCAM expression in mouse models in this manuscript were obtained from the GEO repositories. GSE4648:

https://www.ncbi.nlm.nih.gov/geo/query/acc.cgi?acc=GSE4648 [21], LV from sham and from ischemic/infarcted tissue and the surviving free wall from AMI animals, $15 \mathrm{~min}$ to $48 \mathrm{~h}$ post $\mathrm{AMI}(\mathrm{n}=2$ for each condition). GSE775: https://www.ncbi.nlm.nih.gov/geo/query/ acc.cgi [22], LV from sham and from non-infarcted and infarcted area of AMI mice at $1 \mathrm{~h}, 4 \mathrm{~h}, 24 \mathrm{~h}, 48 \mathrm{~h}, 1$ week, and 8 weeks $(\mathrm{n}=3$ for each condition).

\subsection{Statistical analysis}

Baseline characteristics and patient demographics were compared between ALCAM quartile groups using Kruskal-Wallis tests for continuous variables and Chi-square tests for categorical variables. The association between ALCAM and other biomarkers were assessed by Spearman rank correlation coefficient. Biomarkers were logarithmic transformed when appropriate. The relationship between natural logtransformed ALCAM and baseline characteristics was assessed by multivariable linear models. We calculated geometric means using the antilogarithms of the model-adjusted means (i.e. predicted marginal means), and subsequently compared geometric means between groups 
(e.g. males/females) using ratios.

The unadjusted association between ALCAM quartile groups and clinical outcomes were presented by Kaplan-Meier curves. Cox proportional hazards models were used to investigate the covariate-adjusted association between ALCAM and composite endpoint of CV death, spontaneous MI or stroke, and secondly these components individually. Six models, with incremental addition of co-variates, were used. Model 1 included ALCAM and randomized treatment (ticagrelor or clopidogrel). Model 2 added clinical baseline risk factors; age, gender, body mass index (BMI), diabetes mellitus (DM), chronic kidney disease (CKD), hypertension, smoking status, type of ACS, and history of heart failure (HF), MI, percutaneous coronary intervention (PCI), $\mathrm{CABG}$, stroke, or peripheral artery disease (PAD). Model 3 included all variables from model 2 together with hs-CRP and WBC. Model 4 included all the previous mentioned co-variates, with the addition of cystatin C, a marker of kidney dysfunction. Model 5 included all variables in addition to hs-TnT and NT-proBNP. Model 6 included all variables and growth differentiation factor -15 (GDF-15). All biomarkers were included as continuous variables after logarithmic transformation. The results were presented as the relative hazard for $50 \%$ increase in ALCAM concentration at baseline. The proportional hazards assumption was assessed by visual inspection of Schoenfeld residual plots.
The discriminative value of ALCAM was assessed using C-index. The models with and without ALCAM were compared using likelihood ratio tests.

The effects of ALCAM levels on outcomes in relation to predefined subgroup factors (i.e. randomized treatment, ACS type, invasive/noninvasive in-hospital treatment approach, DM, gender and smoking), were evaluated using Cox proportional hazards models including quartile divided ALCAM levels, the respective subgroup factor and the ALCAM subgroup factor interaction term as independent variables. Two-sided $p$-value of $<0.05$ was considered to be statistically significant and there were no adjustments for multiple comparisons. All statistical analyses were performed with SAS 9.4 (SAS Institute, Cary, NC).

\section{Results}

\subsection{ALCAM in relation to baseline characteristics}

ALCAM concentrations were available in 5156 patients, with a median (Q1-Q3) of $97(80-116) \mathrm{ng} / \mathrm{mL}$. The blood samples for ALCAM analysis were collected $15 \mathrm{~h}(\mathrm{Q} 1-\mathrm{Q} 3:$ 8-21 h) after the index event, i.e., $10 \mathrm{~h}(\mathrm{Q} 1-\mathrm{Q} 3:$ 3-17 h) after admission.

Baseline characteristics by ALCAM quartile divided group are

Table I

Baseline characteristics by quartile divided ALCAM concentrations at baseline.

\begin{tabular}{|c|c|c|c|c|c|}
\hline Characteristic & $\begin{array}{l}\mathrm{Q} 1 \\
<80.2 \mathrm{ng} / \mathrm{mL} \\
\mathrm{n}=1289\end{array}$ & $\begin{array}{l}\mathrm{Q} 2 \\
80.2-96.5 \mathrm{ng} / \mathrm{mL} \\
\mathrm{n}=1289\end{array}$ & $\begin{array}{l}\mathrm{Q} 3 \\
96.5-116.1 \mathrm{ng} / \mathrm{mL} \\
\mathrm{n}=1290\end{array}$ & $\begin{array}{l}\mathrm{Q} 4 \\
>116.1 \mathrm{ng} / \mathrm{mL} \\
\mathrm{n}=1288\end{array}$ & $p$-value \\
\hline Age, yrs & $59(52-67)$ & $61(53-69)$ & $63(55-71)$ & $66(56-73)$ & $<.0001$ \\
\hline Female & $191(14.8 \%)$ & $319(24.7 \%)$ & $462(35.8 \%)$ & $573(44.5 \%)$ & $<.0001$ \\
\hline Weight $\mathrm{kg}$ & $82(73-90)$ & $80(70-90)$ & $80(70-90)$ & $79(69-90)$ & $<.0001$ \\
\hline BMI kg/m ${ }^{2}$ & $27.6(25.0-30.1)$ & $27.5(24.8-30.5)$ & $27.7(25.2-30.7)$ & $27.7(25.0-31.2)$ & 0.0489 \\
\hline \multicolumn{6}{|l|}{ Risk factors a features } \\
\hline Habitual smoker & $557(43.2 \%)$ & $512(39.7 \%)$ & $416(32.2 \%)$ & $413(32.1 \%)$ & $<.0001$ \\
\hline Hypertension & $755(58.6 \%)$ & $844(65.5 \%)$ & $877(68.0 \%)$ & $924(71.7 \%)$ & $<.0001$ \\
\hline Dyslipidemia & $506(39.3 \%)$ & $531(41.2 \%)$ & $576(44.7 \%)$ & $565(43.9 \%)$ & 0.0208 \\
\hline Diabetes mellitus & $222(17.2 \%)$ & $251(19.5 \%)$ & $313(24.3 \%)$ & $361(28.0 \%)$ & $<.0001$ \\
\hline \multicolumn{6}{|l|}{ Medical history } \\
\hline Angina pectoris & $553(42.9 \%)$ & $580(45.0 \%)$ & $634(49.1 \%)$ & $633(49.1 \%)$ & 0.0017 \\
\hline Myocardial infarction & $226(17.5 \%)$ & $242(18.8 \%)$ & $262(20.3 \%)$ & $280(21.7 \%)$ & 0.0419 \\
\hline Congestive heart failure & $41(3.2 \%)$ & $53(4.1 \%)$ & $82(6.4 \%)$ & $120(9.3 \%)$ & $<.0001$ \\
\hline Percutaneous coronary Intervention & $139(10.8 \%)$ & $163(12.6 \%)$ & $159(12.3 \%)$ & $176(13.7 \%)$ & 0.1660 \\
\hline Coronary artery bypass grafting & $61(4.7 \%)$ & $54(4.2 \%)$ & $62(4.8 \%)$ & $79(6.1 \%)$ & 0.1335 \\
\hline Transient ischemic attack & $23(1.8 \%)$ & $30(2.3 \%)$ & $24(1.9 \%)$ & $36(2.8 \%)$ & 0.2662 \\
\hline Non-hemorrhagic stroke & $37(2.9 \%)$ & $43(3.3 \%)$ & $45(3.5 \%)$ & $51(4.0 \%)$ & 0.5004 \\
\hline Peripheral arterial disease & $71(5.5 \%)$ & $90(7.0 \%)$ & $90(7.0 \%)$ & $98(7.6 \%)$ & 0.1818 \\
\hline Chronic kidney disease & $20(1.6 \%)$ & $36(2.8 \%)$ & $43(3.3 \%)$ & $81(6.3 \%)$ & $<.0001$ \\
\hline ST-elevation myocardial infarction & $711(55.2 \%)$ & $592(45.9 \%)$ & $574(44.5 \%)$ & $485(37.7 \%)$ & $<.0001$ \\
\hline GRACE risk score & $133(116-149)$ & $132(116-149)$ & $135(120-151)$ & $138(120-157)$ & $<.0001$ \\
\hline \multicolumn{6}{|l|}{ In-hospital medication } \\
\hline Aspirin & $1274(98.8 \%)$ & $1264(98.1 \%)$ & $1265(98.1 \%)$ & $1270(98.6 \%)$ & 0.2900 \\
\hline Unfractionated heparin & $735(57.0 \%)$ & $707(54.8 \%)$ & $673(52.2 \%)$ & $702(54.5 \%)$ & 0.1043 \\
\hline Low-molecular-weight heparin & $676(52.4 \%)$ & $718(55.7 \%)$ & $676(52.4 \%)$ & $714(55.4 \%)$ & 0.1610 \\
\hline Fondaparinux & $16(1.2 \%)$ & $19(1.5 \%)$ & $21(1.6 \%)$ & $17(1.3 \%)$ & 0.8463 \\
\hline Bivalirudin & $21(1.6 \%)$ & $14(1.1 \%)$ & $16(1.2 \%)$ & $25(1.9 \%)$ & 0.2649 \\
\hline Glycoprotein IIb/IIIa inhibitors & $421(32.7 \%)$ & $367(28.5 \%)$ & $312(24.2 \%)$ & $271(21.0 \%)$ & $<.0001$ \\
\hline Beta-blockers & $1131(87.7 \%)$ & $1135(88.1 \%)$ & $1125(87.2 \%)$ & $1095(85.0 \%)$ & 0.0925 \\
\hline 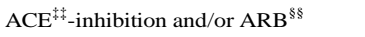 & $1121(87.0 \%)$ & $1096(85.0 \%)$ & $1115(86.4 \%)$ & $1144(88.8 \%)$ & 0.0400 \\
\hline Cholesterol lowering (Statin) & $1238(96.0 \%)$ & $1221(94.7 \%)$ & $1196(92.7 \%)$ & $1174(91.1 \%)$ & $<.0001$ \\
\hline \multicolumn{6}{|l|}{ Biomarkers } \\
\hline Cystatin mg/L median (Q1-Q3) & $0.75(0.62-0.89)$ & $0.79(0.65-0.96)$ & $0.84(0.69-1.02)$ & $0.90(0.73-1.13)$ & $<.0001$ \\
\hline eGFR median (Q1-Q3) & $120(94-120)$ & $112(85-120)$ & $103(78-120)$ & $93(67-120)$ & $<.0001$ \\
\hline Creatinin $\mathrm{mg} / \mathrm{dL}$ & $80(71-88)$ & $80(71-88)$ & $80(71-97)$ & $80(71-97)$ & $<.0001$ \\
\hline CRP mg/L & $3.2(1.4-7.7)$ & $3.4(1.6-8.6)$ & $3.7(1.6-8.8)$ & $3.7(1.8-9.6)$ & 0.0083 \\
\hline GDF- $15 \mathrm{pg} / \mathrm{mL}$ & $1361(1032-1853)$ & $1468(1107-2035)$ & $1551(1155-2218)$ & $1807(1326-2592)$ & $<.0001$ \\
\hline Troponin $\mathrm{T}$ hs ng/L & $167(42-582)$ & $158(39-566)$ & $160(41-511)$ & $152(36-580)$ & 0.5743 \\
\hline NT-proBNP pmol/L & $313(99-842)$ & $389(119-993)$ & $448(149-1311)$ & $560(199-1572)$ & $<.0001$ \\
\hline White blood cells $\times 10^{9}$ cells $/ \mathrm{L}$ & $9.6(7.7-11.9)$ & $9.4(7.4-11.6)$ & $9.3(7.5-11.7)$ & $9.0(7.1-11.2)$ & 0.0002 \\
\hline
\end{tabular}

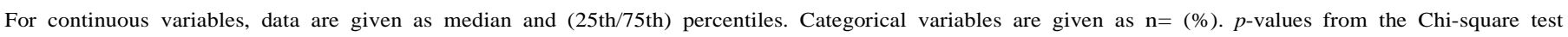
(categorical variables) or Kruskal-Wallis test (continuous variables). 
shown in Table $1(\mathrm{n}=5156)$ and comparison between the current substudy and total PLATO cohort $(\mathrm{n}=18421)$ is shown in Supplemental Table 1. The populations were similar except for more frequent STEMI in the current sub-population. Supplemental Table 2 shows multivariable effects of baseline characteristics on ALCAM at baseline and 1 month. The four strongest determinants of ALCAM at both time-points were female sex, chronic renal disease, age and STEMI. Of note, patients with Q4 ALCAM had a 1.9 times higher history of previous HF compared to Q1.

Of other relevant biomarkers previously determined in PLATO, high ALCAM levels were associate with poorer kidney function (i.e. high cystatin C and lower eGFR), high NT-proBNP and GDF-15 as markers of poorer cardiac function, but not with hsTnT, and with declining WBC counts.

\subsection{ALCAM in relation to circulating cardiac and inflammatory markers}

As shown in Supplemental Table 3, the strongest correlation for ALCAM was with GDF15 and cystatin C, followed by Fibrin D-dimer, NT-proBNP and IL-18. Moreover, ALCAM correlated positively with Pselectin, another cell adhesion molecule reflecting platelet and endothelial cell activation. Furthermore, these associations were present also at 1 month with slightly attenuated coefficients. In contrast, weaker or negative correlations were observed with markers reflecting plaque progression such as CRP, oxLDL, WBC or leukocyte subgroups.

\subsection{ALCAM concentrations during follow up}

Fig. 1 shows ALCAM levels during 6 months of follow-up. ALCAM levels gradually increased from the acute event. Already at discharge, there was a significant increase from baseline (median change 1.5, 25th/75th percentile: $-10.2,14.2 \mathrm{ng} / \mathrm{mL}, p<0.0001)$. ALCAM was further elevated at 1 month (median change $14 \quad[1,30] \mathrm{ng} / \mathrm{mL}$, $p<0.0001$ ) and thereafter remained stable until 6 months (median change $13[-2,29] \mathrm{ng} / \mathrm{mL}, p<0.0001)$ compared with baseline levels. A modest increase in ALCAM was observed in the ticagrelor group compared to the clopidogrel group at 6 months $(112 \mathrm{vs} .110 \mathrm{ng} / \mathrm{mL}$, $p=0.003$ ) (Supplemental Table 4).

\subsection{ALCAM and CV outcomes}

During follow-up, the primary endpoint was observed in 434 (8.4\%) patients (191 CV fatalities, 243 spontaneous MI and 62 stroke events). Restricted cubic spline analysis revealed a relatively linear association between ALCAM levels at baseline and incidence of the primary endpoint (Fig. 2A).

KM rates showed a lower event rate $(6.2 \%)$ for quartile divided group 1 of baseline ALCAM, with similar event rates for quartile divided group $2(6.8 \%)$, an increase in quartile divided group $3(8.8 \%)$ and a markedly higher event rate for quartile divided group $4(11.8 \%$, $p<0.0001$ ). As shown in Table 2, the unadjusted hazard ratio (HR) and $95 \%$ confidence interval, for the primary outcome measures per $50 \%$ increase in baseline ALCAM concentration, was 1.43 (1.25-1.62), $p<0.0001$. This remained significant after adjustment for clinical characteristics, inflammatory (e.g. CRP, WBC) and cardiac (e.g. hsTnT and NTproBNP) biomarkers as well as GDF-15 and cystatin C (1.16 [1.00-1.34], $p=0.043$ ).

The results for individual components of the primary endpoint showed that the association between ALCAM and this endpoint was driven by the effects on CV-death. Restricted cubic spline analysis revealed a somewhat linear association between ALCAM levels at baseline and incidence of CV-death (Fig. 2C), and a gradual increase in event rates was observed from quartile 1 through 4 of $\operatorname{ALCAM}(1.4 \%, 2.9 \%$, $4.2 \%, 6.4 \%)$. This association remained significant also in the fully adjusted analysis (1.45 [1.16-1.82], $p=0.0012$, Table 2). Supplemental Table 5 shows model improvement by addition of
ALCAM at the different steps of adjustment for the primary endpoint and CV-death. Modest gains in the $\mathrm{C}$ index were observed in the more advanced models, but the LR-test demonstrated model improvement. No association of ALCAM with spontaneous MI or stroke was observed (Supplemental Table 6).

There were no interactions between ALCAM levels and outcomes by randomized treatment, type of ACS, in-hospital treatment approach, diabetes, gender or smoking (Supplemental Table 7).

\subsection{ALCAM concentrations and outcomes at 1 month}

As shown in Table 2, the association between high ALCAM levels and the primary endpoint and CV death was also present at 1 month, with a slightly lower HRs and wider CIs. These associations were significant also after adjustment for clinical variables, but were lost upon adjustment with inflammatory markers and further declined with the inclusion of more adjustment variables. No association between ALCAM at 1 month and either sMI or stroke was observed (Supplemental Table 6).

\subsection{Evaluation of ALCAM expression from public databases}

To elucidate the regulation of ALCAM during MI, we finally evaluated the expression of this adhesion molecule in relevant settings from public available databases. In the first study (GSE4648), ALCAM mRNA expression was similar in myocardial tissue obtained from the left ventricular (LV) of sham animals and LV tissue from the surviving free wall and ischemic/infarcted area from MI animals until $12 \mathrm{~h}$ post-MI (Supplemental Fig. 1A). After 12 h, however, ALCAM expression increased and peaked at $24 \mathrm{~h}$ ( 2-fold increase) in LV from the ischemic/ infarcted area as compared with non-infarcted/ischemic LV and LV from sham animals. In another MI mouse study (GSE775), ALCAM mRNA expression in LV was stable in sham animals and in the noninfarcted area, but increased markedly in LV infarcted tissue from $24 \mathrm{~h}$ and peaked at 1 week ( 6-fold increase) followed by a decline to normal levels at 8 weeks (Supplemental Fig. 1B). As mice age generally 25x faster than humans (i.e. 25 times shorter lifespan) [23], these temporal changes in myocardial ALCAM mRNA expression in MI mice corresponds to the systemic increase we observe for ALCAM in PLATO.

\section{Discussion}

In a large, contemporary ACS population treated with dual antiplatelet treatment, serum levels of ALCAM were independently associated with increased risk of the composite of CV death, sMI and stroke. This was mainly driven by an association with CV death, which persisted also after adjustment with multiple clinical, CV and inflammatory biomarkers prognostic for $\mathrm{CV}$ events. A similar, but weaker association

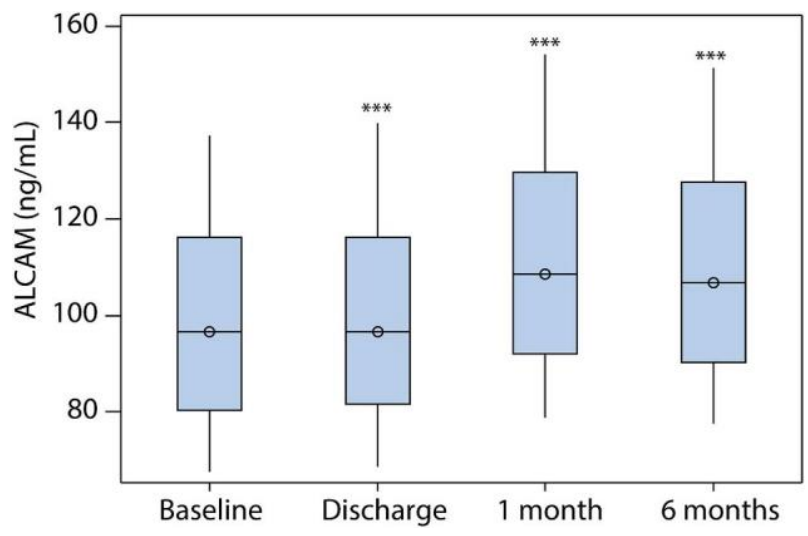

Fig. I. ALCAM levels at baseline and during follow up. $* * * p<0.0001 v s$. baseline. 

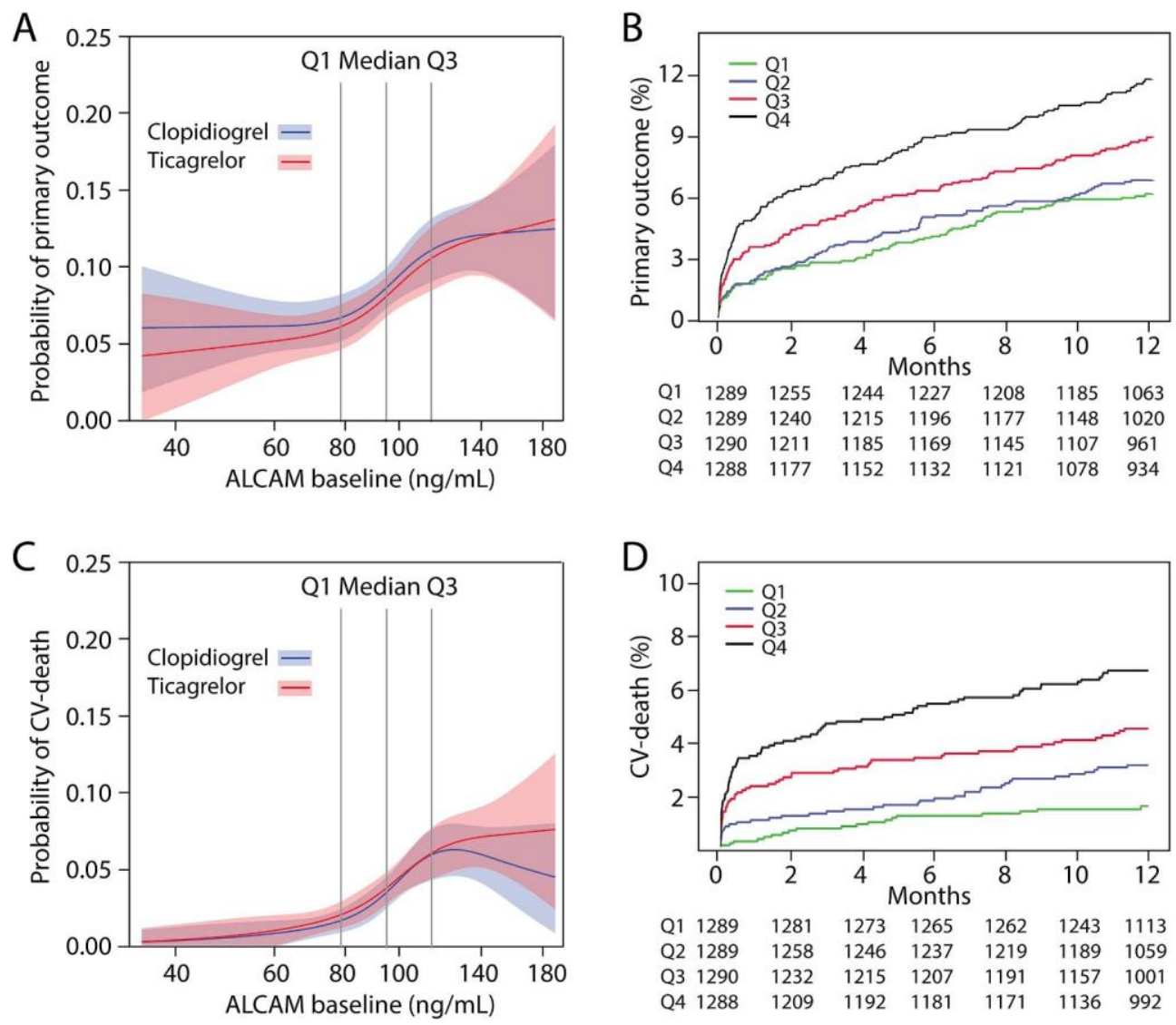

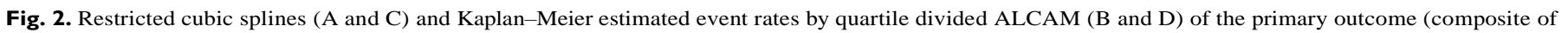
cardiovascular death, spontaneous myocardial infarction, and stroke) and cardiovascular death (CV-death).

of this biomarker with outcome was also seen at 1 month, but not significant in fully adjusted analysis. There was no interaction between ALCAM and the effects of ticagrelor although ALCAM levels were slightly higher during ticagrelor treatment at 6 months follow-up. Data on ALCAM in ACS are scarce, but our study suggests that ALCAM might reflect important pathophysiological processes that are only partly mirrored by other biomarkers, contributing to CV death in ACS patients.

We have previously demonstrated that high serum levels of ALCAM following acute ischemic stroke are associated with all-cause- and in particular CV-mortality during long-term follow-up, also in multivariable analysis including stroke severity, CRP and TnT levels [11]. In the present much larger study in ACS patients, we found a similar association between high admission levels of ALCAM and the primary endpoint (CV death, sMI and stroke). This was mainly driven by a strong association between ALCAM and CV death. There are numerous studies on biomarkers in ACS, but as opposed to the current study, in most cases these markers lose their prognostic value after adjustment for established markers such as CRP, troponins and natriuretic peptides. Notably, in the present study the association of ALCAM and CV-death

Table 2

Associations between continuous (hazard ratios [HRs] per 50\% increase in ALCAM at baseline and 1 month ${ }^{\mathrm{a}}$ and the primary outcome and CV-death.

\begin{tabular}{|c|c|c|c|c|c|c|c|}
\hline \multirow[t]{2}{*}{ Model } & \multirow[t]{2}{*}{ Time } & \multicolumn{3}{|c|}{ Primary (CV-death, spontaneous MI, and stroke) } & \multicolumn{3}{|l|}{ CV-death } \\
\hline & & $\mathrm{N}($ event rate\%) & HR $(95 \% \mathrm{CI})$ & $p$ & $\mathrm{~N}$ (event rate\%) & HR $(95 \% \mathrm{CI})$ & $p$ \\
\hline \multirow[t]{2}{*}{ M1 } & BL & $5156(8.4 \%)$ & $1.43(1.24-1.62)$ & $<.0001$ & $5156(3.7 \%)$ & $2.01(1.66-2.44)$ & $<.0001$ \\
\hline & $1 \mathrm{Mo}$ & $4238(5.7 \%)$ & $1.40(1.16-1.68)$ & 0.0004 & $4238(2.0 \%)$ & $1.94(1.43-2.64)$ & $<.0001$ \\
\hline \multirow[t]{2}{*}{ M2 } & $\mathrm{BL}$ & $5144(8.4 \%)$ & $1.27(1.11-1.46)$ & 0.0004 & $5144(3.7 \%)$ & $1.82(1.48-2.25)$ & $<.0001$ \\
\hline & $1 \mathrm{Mo}$ & $4231(5.7 \%)$ & $1.25(1.03-1.52)$ & 0.0247 & $4231(2.0 \%)$ & $1.71(1.23-2.36)$ & 0.0013 \\
\hline \multirow[t]{2}{*}{ M3 } & BL & $4454(8.4 \%)$ & $1.27(1.10-1.47)$ & 0.0013 & $4454(3.8 \%)$ & $1.68(1.34-2.10)$ & $<.0001$ \\
\hline & $1 \mathrm{Mo}$ & $3061(5.5 \%)$ & $1.21(0.96-1.53)$ & 0.1118 & $3061(1.7 \%)$ & $1.52(0.99-2.32)$ & 0.0553 \\
\hline \multirow[t]{2}{*}{ M4 } & BL & $4453(8.4 \%)$ & $1.21(1.05-1.40)$ & 0.0097 & $4453(3.8 \%)$ & $1.56(1.24-1.96)$ & 0.0001 \\
\hline & $1 \mathrm{Mo}$ & $3061(5.5 \%)$ & $1.12(0.88-1.42)$ & 0.3466 & $3061(1.7 \%)$ & $1.25(0.81-1.94)$ & 0.3162 \\
\hline \multirow[t]{2}{*}{ M5 } & $\mathrm{BL}$ & $4429(8.4 \%)$ & $1.19(1.03-1.238)$ & 0.0203 & $4429(3.8 \%)$ & $1.49(1.18-1.87)$ & 0.0007 \\
\hline & $1 \mathrm{Mo}$ & $3057(5.5 \%)$ & $1.10(0.87-1.40)$ & 0.4285 & $3057(1.7 \%)$ & $1.20(0.77-1.86)$ & 0.4276 \\
\hline \multirow[t]{2}{*}{ M6 } & BL & $4429(8.4 \%)$ & $1.16(1.00-1.34)$ & 0.0430 & $4429(3.8 \%)$ & $1.45(1.16-1.82)$ & 0.0012 \\
\hline & $1 \mathrm{Mo}$ & $3057(5.5 \%)$ & $1.09(0.86-1.38)$ & 0.4575 & $3057(1.7 \%)$ & $1.21(0.78-1.88)$ & 0.3883 \\
\hline
\end{tabular}

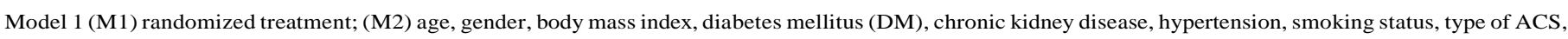

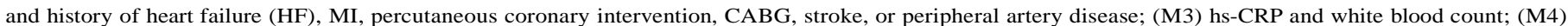
cystatin C; (M5) hs-TnT and NT-proBNP; (M6) GDF-15.

a The 1-month data are for patients who had no CV events (spont. MI or stroke) before the date of the 1-month sample. 
remained significant following the addition of CRP, NT-proBNP, hsTnT, GDF-15 and cystatin $C$ which previously have been shown to display independent associations with CV-death in this study population [15-17,24]. This could indicate that ALCAM reflects activation of pathways not mirrored by more established biomarkers.

Several observations suggest that the enhanced ALCAM levels in ACS patients with poor prognosis could be related to the infl response and myocardial remodeling following acute MI rather than plaque progression and destabilization prior to ACS. First, WBC has been shown to correlate strongly with plaque characteristics in ACS [25] and our finding that ALCAM correlated poorly with WBC or other leukocyte sub-populations, does not support interactions between ALCAM and plaque vulnerability. Second, ALCAM consistently (i.e. at baseline and 1 month) correlated with markers linked to LV remodeling and infl following cardiac injury such as NT-proBNP, GDF15, IL-18 and P-selectin, and less with markers refl immediate myocardial damage (TnT) or atherogenic and metabolic risk (e.g. CRP, WBC, oxLDL). Third, a markedly higher proportion of patients with high ALCAM levels (i.e. Q4), compared to low ALCAM, had a history of HF, further supporting a link to cardiac dysfunction. Fourth, ALCAM was associated with CV death, but not MI or Stroke suggesting a stronger correlation with cardiac dysfunction rather than atherosclerotic progression. Taken together, we speculate that increased serum ALCAM levels could, at least partly, refl myocardial infl in ACS.

ALCAM levels increased at 1-month follow-up and remained at the same levels until 6 months. As the inflammatory response increases during post-infarction cardiac remodeling, the higher levels of ALCAM during follow-up may suggest a role for ALCAM in this process. The persistently raised myocardial ALCAM expression following experimental MI in mice, corresponding to the time frame in the current study, may further support such a notion. Although the impact of ALCAM in this setting is unknown, the association between high ALCAM levels and CV-death at both baseline and 1 month may suggest a detrimental effect. ALCAM is expressed by activated monocytes and macrophages and while the inflammatory response is integral to the healing process, an excessive, prolonged infiltration of inflammatory cells in the infarcted area is harmful and may contribute to tissue destruction, interstitial fibrosis, cardiac dysfunction, and adverse LV remodeling [26,27], thus resulting in poor clinical outcome [28]. Indeed, in vivo silencing of endothelial CAMs impaired post-MI monocyte recruitment to the remote myocardium and preserved ejection fraction [29]. Furthermore, through interaction with CD6 on T cells, ALCAM promotes an inflammatory phenotype and is required for optimal $\mathrm{T}$ cell activation, which could contribute to inflammation and tissue damage through $\mathrm{T}$ cell-mediated mechanisms [7,8]. It is tempting to hypothesize that a similar mechanism could be operating within the myocardium following MI. The role and function of ALCAM during post-MI remodeling deserves further attention in mechanistic studies.

The reason for the higher levels of ALCAM at 6 months follow-up during ticagrelor maintenance therapy are at present not clear but some possibilities may be relevant. First, we have previously reported from the PLATO study that patients treated with ticagrelor have elevated levels of some inflammatory markers such as IL-6 as compared with those receiving clopidogrel [18]. Moreover, the potential involvement of ALCAM in maladaptive remodeling may not necessarily involve antiapoptotic affects during the acute phase, but rather a hypertrophic and maladaptive effect in the post-MI remodeling phase. Whatever the mechanisms, it is tempting to speculate that whereas ticagrelor improves outcome following ACS, additional improvement could be obtained by targeting pathways that are not modified by this treatment option such as ALCAM related pathways.

\subsection{Limitations}

The generalizability of this study might be limited to patients meeting the inclusion criteria for PLATO. However, the patients included in PLATO represent a broad range of ACS patients fairly well corresponding to the real-life situation. We evaluated interactions between ALCAM levels and outcomes by several subgroups (e.g., diabetes, type of ACS) but we cannot exclude that other comorbidities could influence our results. Thus, ALCAM has been shown to be suitable as a prognostic marker for different types of cancer [30], which, however, has a non-negligible prevalence and impact on outcome in ACS patients [31]. Beyond NT-proBNP and demographic data on previous HF, our study lacks objective measures concerning LV systolic function, which would allow us to mare closely assess the association between ALCAM and cardiac dysfunction. We speculate on the role of ALCAM in ACS based on correlation analysis with established markers reflecting specific cell numbers or pathophysiological processes, but correlations do not necessarily imply any causal relationship and the mechanisms and role of ALCAM in underlying processes during post-MI remodeling will have to be assessed in mechanistic studies. Finally, the prognostic value of ALCAM and other variables might be different in relation to longer follow-up.

\subsection{Conclusions}

In conclusion, in patients with ACS treated with dual antiplatelet treatment, we observed an independent association between admission levels of ALCAM and risk of CV-death. Although the clinical benefit and utilization of ALCAM as a viable biomarker in patients with ACS on optimal antithrombotic therapy could be promising, this should be assessed in independent studies. Based on the strong association with $\mathrm{CV}$ death, the role of ALCAM and the associated underlying processes during post-MI remodeling merits further investigation.

\section{Financial support}

The PLATO study was funded by AstraZeneca. Support for the analyses, interpretation of results and preparation of the manuscript was provided through funds to the Uppsala Clinical Research Center as part of the Clinical Study Agreement, and provided by a grant from the Swedish Strategic Research Foundation. Roche Diagnostics, Rotkreuz, Switzerland supported the research by providing GDF-15 assay free of charge. The authors are entirely responsible for the design and conduct of this study; all study analyses, the drafting and editing of the article and its final contents.

\section{Author contributions}

TU, A $\AA$, SKJ, MB, AS, FK, PA and LW contributed to the conception and design of the study; TU and AEM contributed to the acquisition of data; $\mathrm{A} \AA$, TG, MB, PA, FK and LW contributed to analysis and interpretation of data; TU, A $\AA, P A, M B$ and LW contributed to drafting the article. All authors contributed to critical revision for important intellectual content and given final approval of the version to be submitted.

\section{Declaration of competing interest}

TU, AEM, PA: nothing to report.

A ̊: institutional research grant and speakers fee from AstraZeneca; institutional research grant from Roche Diagnostics, all are considered significant. TG, MB: institutional research grants from AstraZeneca, considered significant. RCB: scientific advisory board member for Janssen, Ionis Pharmaceuticals and AstraZeneca; safety review committee member for Portola, all are considered modest.AH: reports being an employee of AstraZeneca, considered significant. SKJ: institutional research grant, honoraria and consultant/advisory board fee from AstraZeneca, considered significant; institutional research grant and consultant/advisory board fee from Medtronic, considered significant; institutional research grants and honoraria from The Medicines 
Company; consultant/advisory board fees from Janssen, Bayer, all are considered modest. AS: institutional research grants from AstraZeneca, Boehringer Ingelheim, Bristol-Myers Squibb/Pfizer and GlaxoSmithKline, all are considered significant.

RFS: institutional research grants, consultancy fees, and honoraria from AstraZeneca, considered significant; Institutional research grants and consultancy fees from PlaqueTec, considered significant; consultancy fees from Aspen, Avacta, Bayer, Bristol-Myers Squibb/Pfizer, Novartis, The Medicines Company and ThermoFisher Scientific, all are considered modest. FK: consultancy fees/honoraria for lectures, advisory board membership, and fee for research work outside the submitted from AstraZeneca; advisory board membership and consultancy fees from Merck \& Co, all are considered modest. LW: institutional research grants, consultancy fees, lecture fees, and travel support from Bristol-Myers Squibb/Pfizer, AstraZeneca, GlaxoSmithKline, Boehringer Ingelheim, all are considered significant; institutional research grants from Merck \& Co, Roche Diagnostics, considered significant; consultancy fees from Abbott, considered modest; holds two patents involving GDF-15.

\section{Appendix A. Supplementary data}

Supplementary data to this article can be found online at https:// doi.org/10.1016/j.atherosclerosis.2019.11.031.

\section{References}

[1] L. Fang, X.L. Moore, A.M. Dart, L.M. Wang, Systemic inflammatory response following acute myocardial infarction, J. Geriatr. Cardiol.: JGC 12 (2015) 305-312.

[2] N.T. Mulvihill, J.B. Foley, P. Crean, M. Walsh, Prediction of cardiovascular risk using soluble cell adhesion molecules, Eur. Heart J. 23 (2002) 1569-1574.

[3] S. Blankenberg, S. Barbaux, L. Tiret, Adhesion molecules and atherosclerosis, Atherosclerosis 170 (2003) 191-203.

[4] G.S. Hillis, C. Terregino, P. Taggart, et al., Elevated soluble P-selectin levels are associated with an increased risk of early adverse events in patients with presumed myocardial ischemia, Am. Heart J. 143 (2002) 235-241.

[5] M. Hartford, O. Wiklund, L. Mattsson Hulten, et al., C-reactive protein, interleukin6 , secretory phospholipase A2 group IIA and intercellular adhesion molecule-1 in the prediction of late outcome events after acute coronary syndromes, J. Intern. Med. 262 (2007) 526-536.

[6] A.S. Postadzhiyan, A.V. Tzontcheva, I. Kehayov, B. Finkov, Circulating soluble adhesion molecules ICAM- 1 and VCAM- 1 and their association with clinical outcome, troponin $\mathrm{T}$ and $\mathrm{C}$-reactive protein in patients with acute coronary syndromes, Clin. Biochem. 41 (2008) 126-133.

[7] M.A. Bowen, D.D. Patel, X. Li, et al., Cloning, mapping, and characterization of activated leukocyte-cell adhesion molecule (ALCAM), a CD6 ligand, J. Exp. Med. 181 (1995) 2213-2220.

[8] N.J. Hassan, A.N. Barclay, M.H. Brown, Frontline: optimal T cell activation requires the engagement of CD6 and CD166, Eur. J. Immunol. 34 (2004) 930-940.

[9] B. Lin, J. Kim, Y. Li, et al., High-purity enrichment of functional cardiovascular cells from human iPS cells, Cardiovasc. Res. 95 (2012) 327-335.

[10] R. Cayrol, K. Wosik, J.L. Berard, et al., Activated leukocyte cell adhesion molecule promotes leukocyte trafficking into the central nervous system, Nat. Immunol. 9 (2008) 137-145.

[11] L. Smedbakken, J.K. Jensen, J. Hallen, et al., Activated leukocyte cell adhesion molecule and prognosis in acute ischemic stroke, Stroke 42 (2011) 2453-2458.
[12] S. James, A. Akerblom, C.P. Cannon, et al., Comparison of ticagrelor, the first reversible oral $\mathrm{P} 2 \mathrm{Y}(12)$ receptor antagonist, with clopidogrel in patients with acute coronary syndromes: rationale, design, and baseline characteristics of the PLATelet inhibition and patient Outcomes (PLATO) trial, Am. Heart J. 157 (2009) 599-605.

[13] L. Wallentin, R.C. Becker, A. Budaj, et al., Ticagrelor versus clopidogrel in patients with acute coronary syndromes, N. Engl. J. Med. 361 (2009) 1045-1057.

[14] K. Thygesen, J.S. Alpert, A.S. Jaffe, et al., Third universal definition of myocardial infarction, Eur. Heart J. 33 (2012) 2551-2567.

[15] E. Hagstrom, S.K. James, M. Bertilsson, et al., Growth differentiation factor-15 level predicts major bleeding and cardiovascular events in patients with acute coronary syndromes: results from the PLATO study, Eur. Heart J. 37 (2016) 1325-1333.

[16] L. Wallentin, D. Lindholm, A. Siegbahn, et al., Biomarkers in relation to the effects of ticagrelor in comparison with clopidogrel in non-ST-elevation acute coronary syndrome patients managed with or without in-hospital revascularization: a substudy from the Prospective Randomized Platelet Inhibition and Patient Outcomes (PLATO) trial, Circulation 129 (2014) 293-303.

[17] M.A. Velders, L. Wallentin, R.C. Becker, et al., Biomarkers for risk stratification of patients with ST-elevation myocardial infarction treated with primary percutaneous coronary intervention: insights from the Platelet Inhibition and Patient Outcomes trial, Am. Heart J. 169 (2015) 879-889 e7.

[18] R.F. Storey, S.K. James, A. Siegbahn, et al., Lower mortality following pulmonary adverse events and sepsis with ticagrelor compared to clopidogrel in the PLATO study, Platelets 25 (2014) 517-525.

[19] A. Johansson, N. Eriksson, R.C. Becker, et al., NLRC4 inflammasome is an important regulator of interleukin-18 levels in patients with acute coronary syndromes: genome-wide association study in the PLATelet inhibition and patient outcomes trial (PLATO), Circ Cardiovasc Genet 8 (2015) 498-506.

[20] A. Lowenstern, R.F. Storey, M. Neely, et al., Platelet-related biomarkers and their response to inhibition with aspirin and $\mathrm{p} 2 \mathrm{y} 12$-receptor antagonists in patients with acute coronary syndrome, J. Thromb. Thrombolysis 44 (2017) 145-153.

[21] M.H. Harpster, S. Bandyopadhyay, D.P. Thomas, et al., Earliest changes in the left ventricular transcriptome postmyocardial infarction, Mamm. Genome : Off. J. Int. Mamm. Genome Soc. 17 (2006) 701-715.

[22] O. Tarnavski, J.R. McMullen, M. Schinke, Q. Nie, S. Kong, S. Izumo, Mouse cardiac surgery: comprehensive techniques for the generation of mouse models of human diseases and their application for genomic studies, Physiol. Genom. 16 (2004) 349-360.

[23] K. Flurkey, J.M. Currer, The Mouse in Aging Research, American College Laboratory Animal Medicine (Elsevier), Burlington, MA, 2007, pp. 637-672.

[24] A. Akerblom, L. Wallentin, A. Siegbahn, et al., Cystatin C and estimated glomerular filtration rate as predictors for adverse outcome in patients with ST-elevation and non-ST-elevation acute coronary syndromes: results from the Platelet Inhibition and Patient Outcomes study, Clin. Chem. 58 (2012) 190-199.

[25] O.C. Raffel, G.J. Tearney, D.D. Gauthier, E.F. Halpern, B.E. Bouma, I.K. Jang, Relationship between a systemic inflammatory marker, plaque inflammation, and plaque characteristics determined by intravascular optical coherence tomography, Arterioscler. Thromb. Vasc. Biol. 27 (2007) 1820-1827.

[26] J. Liu, H. Wang, J. Li, Inflammation and inflammatory cells in myocardial infarction and reperfusion injury: a double-edged sword, Clin. Med. Insights Cardiol. 10 (2016) 79-84.

[27] P. Libby, M. Nahrendorf, F.K. Swirski, Leukocytes link local and systemic inflammation in ischemic cardiovascular disease: an expanded "cardiovascular continuum", J. Am. Coll. Cardiol. 67 (2016) 1091-1103.

[28] N.G. Frangogiannis, The immune system and cardiac repair, Pharmacol. Res. 58 (2008) $88-111$

[29] H.B. Sager, M. Hulsmans, K.J. Lavine, et al., Proliferation and recruitment contribute to myocardial macrophage expansion in chronic heart failure, Circ. Res. 119 (2016) 853-864.

[30] U.H. Weidle, D. Eggle, S. Klostermann, G.W. Swart, ALCAM/CD166: cancer-related issues, CANCER GENOMICS PROTEOMICS 7 (2010) 231-243.

[31] M. Iannaccone, F. D'Ascenzo, P. Vadala, et al., Prevalence and outcome of patients with cancer and acute coronary syndrome undergoing percutaneous coronary intervention: a BleeMACS substudy, Eur. Heart J. Acute Cardiovasc. Care (2017) 2048872617706501. 